\title{
Sensor Fusion based on Complementary Algorithms using MEMS IMU
}

\author{
Dung Duong Quoc ${ }^{1 *}$, Jinwei Sun ${ }^{1}$, Van Nhu Le ${ }^{2}$ and Nguyen Ngoc Tan ${ }^{3}$ \\ ${ }^{1}$ Dept. of Automatic test and control, Harbin Institute of Technology, Harbin 150001, \\ China \\ ${ }^{2}$ Research center for Space Optics Engineering, Harbin Institute of Technology, \\ China \\ ${ }^{3}$ CMC Telecom Company, Pham Hung street, Caugiay district, Hanoi, Vietnam \\ *Corresponding author: Dung Duong Quoc, Email: quocdung_mta@yahoo.com
}

\begin{abstract}
Conventional attitude/orientation estimating filters are generally complex demanding excessive computational burden. This instigates the requirement for a computationally simple yet sufficiently precise algorithm for applications where computational complexity is of prime import. A relatively simple, robust and equally efficient technique in this regard is the development of complementary filters. Gradient Descent based Complementary Algorithm (GDCA) and Explicit Complementary Clgorithm (ECA) are the latest advancement in complementary filters applicable to low cost, low power MEMS based inertial measurement units (IMUs) employing quaternion. These fixed gain estimators employ gyroscope triad for high frequency estimation and accelerometer triad for low frequency attitude estimation. This paper appraises the performance of GDCA and ECA. Both simulation and experimental results are presented for comparative analysis. Simulated data was generated in MATLAB for known orientation in term of Euler angles to validate the filters performance whereas for practical implementation of different scenarios, MEMS based MPU6050 IMU was employed. As only IMU is employed without aided sensory system, the mandate of this research is limited to attitude estimation in terms of Euler roll and pitch angles. Roll of the adjustable filter gains is also assessed for a range of values.
\end{abstract}

Keywords: MEMS, IMU, Attitude Estimation, INS, Filtering Algorithms

\section{Introduction}

The introduction of MEMS based IMU dramatically reduced the size of the sensory set to chip size along with decrease in cost and power utilization. Still in developing phase with inherited noise issues, MEMS IMU consisting of mutually orthogonal triad of gyroscopes and triad of accelerometers provides a complete solution for applications of orientation estimation [16].

Orientation and attitude estimation is extensively researched area over decades [1-15]. The Applications of attitude estimation are wide and include aerial (UAV), land moving (robot) and submersed/underwater (UUV/ROV) [1-4]. Generally, two categories of sensors are used; internal also known as dead reckoning (for example IMU) and external; sometimes known as aided (for example GPS). Theoretically, single integration of the accelerometer data produces velocity and double integration provides position estimation. However, in actual practice this is not valid due to the non-linearity, biases and noise present in the stand alone INS, [6]. Dead reckoning sensors accumulate errors with the passage of time whereas external sensors 
provide output at low frequency resulting in need for aided-INS system so that the two sensory systems are fused to provide accurate estimations [5, 6]. GPS, Depth and heading sensors, APS, DVL etc. are examples of aided sensors employed to compensate errors in position and attitude estimation of internal sensors. Kalman filtering based estimation techniques are usually employed in this regard and are considered a de-facto standard. GPS aided INS, APS aided INS, Vision based INS, LVS aided IMU and DVL aided IMU are some proven algorithms for aerial, robotics and underwater applications using Kalman filter [6][13].

As mentioned, usually Kalman based algorithms are employed to fuse all the installed sensors measurements [10]. The problem associated with Kalman based algorithms is the demand for the computational complexity which may be computationally expensive for some applications [15]. Particle filter employed in this regard is another example which is even more demanding in computationally complexity perspective $[15,17]$. The fuzzy logic based technique has been devised to deal with the attitude estimation problem with relatively less computational burden but applications are limited [22]. In recent years, the extensive search for simple and sufficiently effective filtering techniques resulted in development of both linear and non-linear constant gain based complimentary algorithms. Complementary filters employ low frequency estimation based on accelerometers measurements and high frequency estimation based on gyroscopes measurements and then fuse them together [15-21]. The explicit complementary algorithm (ECA) and gradient descent based complementary algorithms (GDCA) are the recently developed and successfully implemented techniques which have been proven to tender efficient performance requiring little computational cost. Both algorithms make use of quaternion representation for orientation estimation based on IMU measurements. This paper presents comparative analysis of ECA and GDCA based on MEMS IMU measurements for different scenarios.

The paper is organized in five sections followed by conclusion. Section II provides an overview regarding orientation problem. Section III presents the general understanding of the explicit and gradient descent based complementary algorithms. Section IV provides comparative analysis of the two techniques based on Matlab simulation data. Section V presents the same using MEMS based IMU data; discussion and comparative analysis part followed by the conclusion.

\section{MEMS IMU Modeling}

For position and attitude estimation problem, usually the Euler angles (roll, pitch, and yaw) representation is employed. However, error in roll and yaw estimations occur as soon as pitch reaches $90^{\circ}$. This is called gimbal lock. To avoid this situation, quaternion based solution is preferred which is easily interchangeable to Euler angles. Regarding orientation and attitude problem, two frames of reference are used; the term body frame or sensor reference is referred to the measurement taken by the sensor directly whereas earth reference or inertial frame means North-East-Down (NED). Inertial Measurement Unit (IMU) is a chip size unit comprised of triad of accelerometers and gyros. MEMS based IMU's gyroscopes and accelerometers can be modeled as follows:

\subsection{MEMS IMU Gyroscope Model}

Gyroscopes compute the angular velocity but the main concern is the bias issue. For MEMS based IMU, three gyroscopes orthogonally installed measure angular velocity in $\mathrm{x}, \mathrm{y}$, $\mathrm{z}$ directions. Like all the sensors measuring a specific quantity, the gyroscope measurements include noises and biases. Hence a gyroscope can be modeled as: 


$$
\Omega_{g}^{b}(t)=\Omega(t)+s_{g}(t)+n_{g}(t)
$$

Where $\Omega_{g}^{b}(t)$ is gyroscope reading in body coordinates, $\Omega(t)$ is actual angular velocity, is $s_{g}(t)$ the gyroscope bias and $n_{g}(i s)$ the associated noise in the gyroscope measurements.

\subsection{MEMS IMU Accelerometer Model}

In theory, accelerometer measures acceleration only. In actual case, however, accelerometer measures the instantaneous linear acceleration along with the gravitational acceleration field with some added bias and noise which can be modeled as:

$$
R(t)=\dot{v}-g+s_{a}(t)+n_{a}(t)
$$

Here, $\dot{v}$ represents instantaneous linear acceleration $g$ is for gravitational acceleration, $s_{a}(t)$ and $n_{a}(t)$ show accelerometer bias and noise respectively.

\section{Attitude Estimation Algorithms}

Orientation and attitude estimation based on low frequency accelerometer measurements are highly reliable where as estimation based on high frequency gyroscope are accurate. This fact is exploited by complementary algorithms. The two recently developed attitude estimators in focus are detailed below

\subsection{Explicit Complementary Algorithm (ECA)}

Algorithms based on complementary filtering combine accelerometer and gyroscope measurements for orientation estimation in a way so that estimation based on accelerometer measurements are low passed where as high-pass filtering is applied on estimation based on gyroscope output [15]. Explicit complementary algorithm is a nonlinear fixed gain complementary filter employing quaternion. ECA algorithm in quaternion form combines inertial direction measurement ( $\bar{v}$, provided by accelerometers) and angular velocity $\left(\Omega^{b}\right.$, measured from gyroscopes). The filter is equipped with two adjustable parameters like proportional $\left(K_{p}\right)$ and Integral gain $\left(_{K_{l}}\right)$ for fine tuning. Further Details of the algorithm can be found in [20].

The algorithm can be summarized as following:

The algorithm can be summarized as following:

1. Initialization: At this stage, parameters like sampling rate, proportional and integral gain and initial value of attitude as $\left.\left[\begin{array}{llll}1 & 0 & 0 & 0\end{array}\right]\right)$ in quaternion form are defined.

2. Data preprocessing: MEMS based IMU tri-accelerometers data is normalized

3. Estimating Gravity direction: At this stage, to debias gyro data, error is to be worked out by cross multiplying measured inertial gravitational direction $(\bar{v}$, as provided by triaccelerometers) and estimated gravitational direction $(\hat{v})$. The estimated gravitational direction is computed at this stage as:

$$
\hat{v}=\left[\begin{array}{l}
2\left(\hat{q}_{2} \hat{q}_{4}+\hat{q}_{1} \hat{q}_{3}\right) \\
2\left(\hat{q}_{3} \hat{q}_{4}+\hat{q}_{1} \hat{q}_{2}\right) \\
\hat{q}_{1}^{2}-\hat{q}_{2}^{2}-\hat{q}_{3}^{2}+\hat{q}_{4}^{2}
\end{array}\right]
$$

Here, $\hat{q}_{x}$ shows quaternion parameters. 
4. Cross multiplication: At cross multiplication stage, error is calculated by cross multiplying normalized accelerometer data and estimated direction of gravity (as provided by previous step) as:

$$
e=\bar{v} \times \hat{v}
$$

5. Debias gyroscope rate: Now is the time to debias gyroscope measurements so that gyro rate can be employed for attitude estimation by simple integration by applying feedback terms as:

$$
\bar{\Omega}^{b}=\Omega^{b}+K_{p} e+K_{I} \int e
$$

Some suitable values of adjustable parameters $K_{p}$ and $K_{I}$ are provided here.

6. Update rate: compute rate of change of quaternion as:

$$
\dot{\hat{q}}=\frac{1}{2} \hat{q} \otimes p\left(\bar{\Omega}^{b}\right)
$$

Where

$\hat{q}=$ Estimated normalized quaternion at one step previous time $(\mathrm{t}-1)$, $p\left(\bar{\Omega}^{b}\right)=\left(0, \bar{\Omega}^{b}\right)$ and $\otimes$ is quaternion product operator.

7. Estimate updated attitude: Integrate to yield estimated attitude in quaternion and normalize. At this stage, attitude in Euler angles can also be computed.

For the next iteration, the algorithm is repeated from Data Processing stage.

\subsection{Gradient Descent Complementary Algorithm (GDCA)}

GDCA is also nonlinear complementary filtering scheme with single adjustable parameter $\beta$. IMU tri-gyroscopes measuring angular rate can be shown as:

$$
\Omega^{b}=\left[\begin{array}{llll}
0 & \omega_{x}^{b} & \omega_{y}^{b} & \omega_{z}^{b}
\end{array}\right]
$$

The rate of change of angular rate in quaternion representation can be represented as:

$$
\dot{q}_{(\omega, t)}^{b}=\frac{1}{2} \hat{q}^{b} \otimes \Omega^{b}
$$

For orientation estimation, accelerometer measurement is used to debias estimation based on gyroscope data. Orientation estimation is performed using equation (8) and (one step) previous estimation in quaternion as below [16]:

$$
q_{(e s t, t)}^{b}=\hat{q}_{(e s t, t-1)}^{b}+\dot{q}_{(e s t, t)}^{b} \Delta t
$$

Here,

$$
\begin{gathered}
\dot{q}_{(e s t, t)}^{b}=\dot{q}_{(\omega, t)}^{b}-\beta \frac{\nabla f}{\|\nabla f\|} \\
\nabla f=J_{g}^{T}\left(\hat{q}^{b}\right) f_{g}\left(\hat{q}^{b}, \hat{R}^{b}\right)
\end{gathered}
$$




$$
\begin{gathered}
f_{g}\left(\hat{q}^{b}, \hat{R}^{b}\right)=\left[\begin{array}{l}
2\left(q_{2} q_{4}-q_{1} q_{3}\right)-R_{x} \\
2\left(q_{1} q_{2}+q_{3} q_{4}\right)-R_{y} \\
2\left(0.5-q_{2}^{2}-q_{3}^{2}\right)-R_{z}
\end{array}\right] \\
J_{g}\left(\hat{q}^{b}\right)=\left[\begin{array}{rrrr}
-2 q_{3} & 2 q_{4}-2 q_{1} & 2 q_{2} \\
2 q_{2} & 2 q_{1} & 2 q_{4} & 2 q_{3} \\
0 & -4 q_{2} & -4 q_{3} & 0
\end{array}\right]
\end{gathered}
$$

The terms are explained in the following stepwise detailed algorithm:

1. Initialization: Assume initial quaternion as $q=\left[\begin{array}{llll}1 & 0 & 0 & 0\end{array}\right]$, specify sampling time and the adjustable gain $\beta$ value.

2. Normalization step: Normalize IMU tri-accelerometer measurements

3. G.D. Algorithm: gradient descent algorithm to compute using equation (11)

4. Rate quaternion correction: At this stage, correction is made in rate quaternion using equation (10).

5. Final orientation estimation: Finally Integration is done to estimate orientation in quaternion by equation (9) where $\hat{q}_{(e s t, t-1)}^{b}$ is the normalize quaternion estimated at time $t-1 q_{(e s t, t)}^{q^{b}}$ from previous step and $\Delta t$ is sampling time.

6. Quaternion Normalization: Finally, Normalize the estimated quaternion is normalized and can then be interchanged to Euler angles representation.

Next Iteration: Algorithm is repeated from stage 2 for next iteration

\section{Simulation Results}

In this paper unaided INS is considered for comparative analysis, so only roll and pitch are observable. For simulation, Matlab environment was used for known orientations with respect to time in terms of roll and pitch Euler angles. Following equations were used to generate simulated data for MEMS IMU's tri-accelerometers [23]:

$$
\begin{array}{r}
\tan \phi_{x y z}=\left(\frac{R_{y}}{R_{z}}\right) \\
\tan \theta_{x y z}=\frac{-R_{x}}{\sqrt{R_{y}^{2}+R_{z}^{2}}} \\
R_{x}^{2}+R_{y}^{2}+R_{z}^{2}=1
\end{array}
$$

Here, $\phi, \theta$ represent roll and pitch angle respectively in radians, $R$ represents accelerometer data in three dimensions.

As the two complementary algorithms are equipped with adjustable filter gains, so the first issue is to select appropriate fixed filter gain values (tunable parameters, $K_{p}, K_{i}$ for ECA and $\beta$ for GDCA). For tunable parameters, $K_{p}, K_{i}$ (proportional and integral gain respectively) for ECA, usually the values are well below unity such that the value of $K_{p}$ is selected 10 to 100 times that of $K_{i}$ whereas the value of $K_{i}$ is as low as 0.002 [20]. For GDCA, the appropriate value of fixed gain $\beta$ is in the range 0.03 to 0.05 [16]. For a system under high dynamics, the fixed gain may be made dynamic for high accuracy. 


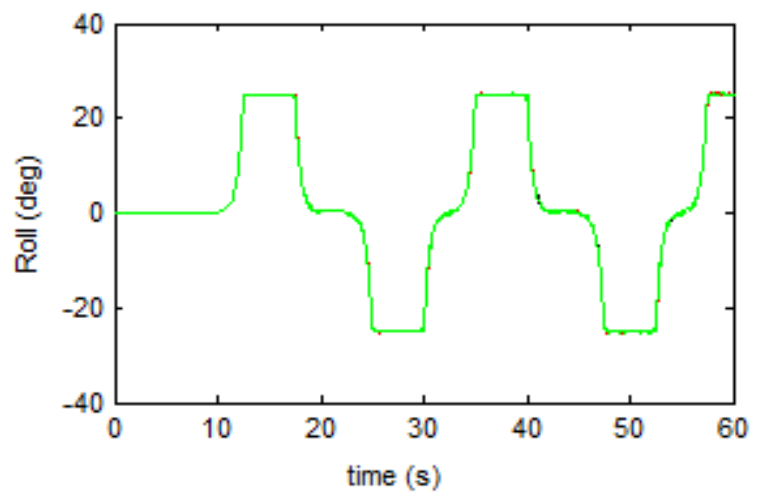

(a)

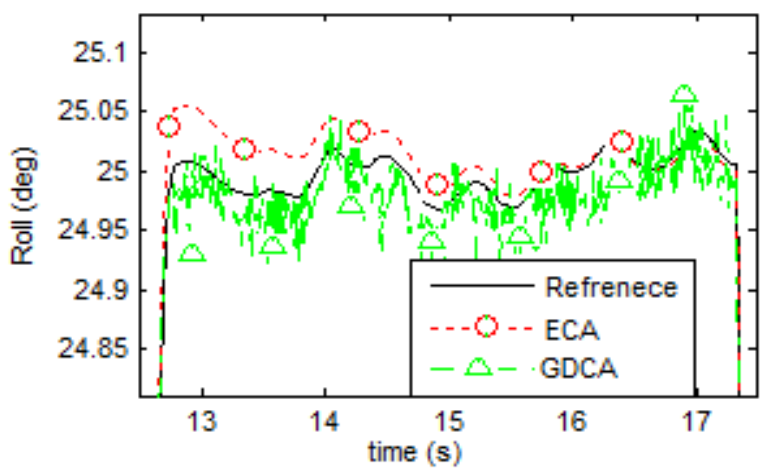

(b)

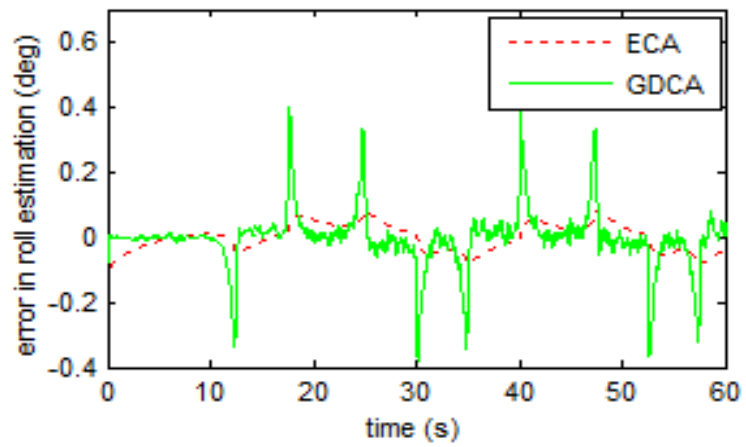

(c)

\section{Figure 1. Roll Estimation from Simulated Data, (a) Roll Euler Angle Estimation} by ECA \& GDCA, (b) Zoom - In View, (c) Error in Roll Estimation

Figure 1 shows the roll Euler angle estimation from Matlab simulated data using ECA and GDCA .Constant values of filter gains were selected such that $K_{i}=0.02 ; K_{p}=0.3$ for ECA and $\beta=0.05$. Figure 2 depicts pitch Euler angle estimation for the same fixed parameters. It is evident from these results that both fixed gain complementary algorithms closely follow the reference orientation (showing that these fixed gain values are appropriate at most of the time). Among the two, ECA estimations are a little bit more accurate and steady as compared to GDCA estimations. 


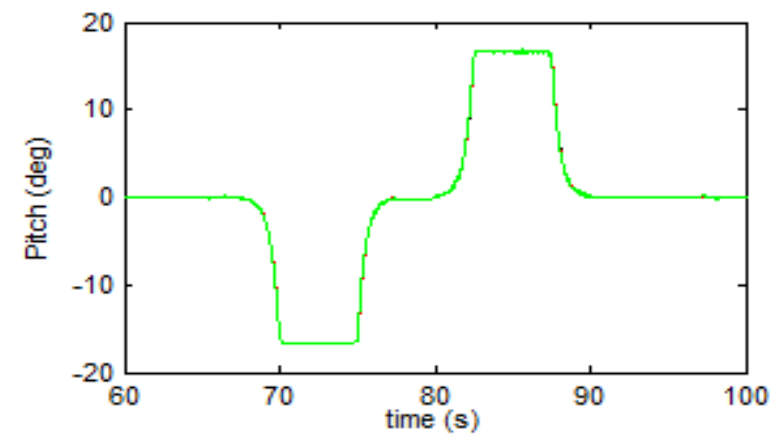

(a)

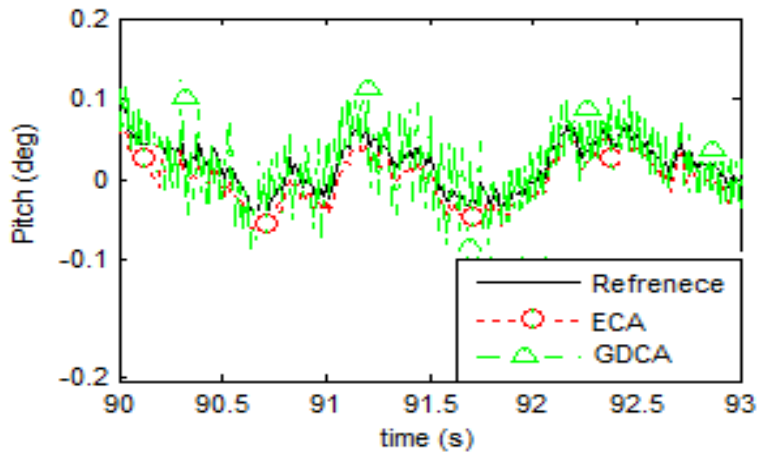

(b)

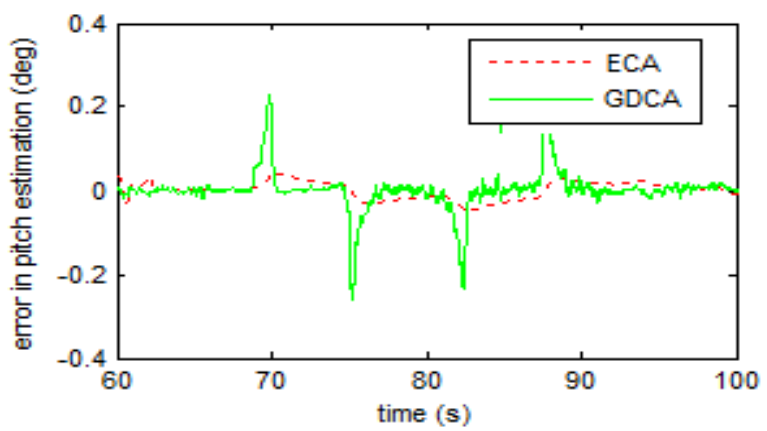

(c)

\section{Figure 2. Pitch Estimation from Simulated Data, (a) Pitch Euler Angle Estimation by ECA \& GDCA, (b) Zoom - In View, (c) Error in Pitch Estimation}

\section{Experimental Results and Discussion}

Experiments were conducted using MEMS based IMU; MPU-6050 IMU (Inertial Measurement Unit) at sampling frequency of $100 \mathrm{~Hz}$; STM32 as interface to CPU. As only comparison of the two complementary algorithms (ECA \& GDCA) was intended, hence IMU was tilted around $\mathrm{X}$ and $\mathrm{Y}$ axis with hand. Also as only IMU measurements were processed without any aided sensor, so only roll and pitch estimations were used for comparative analysis. The adjustable parameters (filter gains) were kept fixed and same as in previous section (For ECA, filter gain values $K_{p}=0.3$ (proportional gain) and $K_{i}=0.02$ (integral gain) whereas $\beta=0.05$ was chosen for GDCA).

First the IMU was kept at stand still for 50 second, tilted for a roll angle in the proximity of 30 degrees, for 80 seconds and then back to original position for next 50 seconds. Same was repeated in the opposite direction for a little short duration. The 
data thus generated from IMU was then processed through both algorithms (ECA and GDCA) in Matlab and again identical results were achieved as shown in Figure 4, 5.

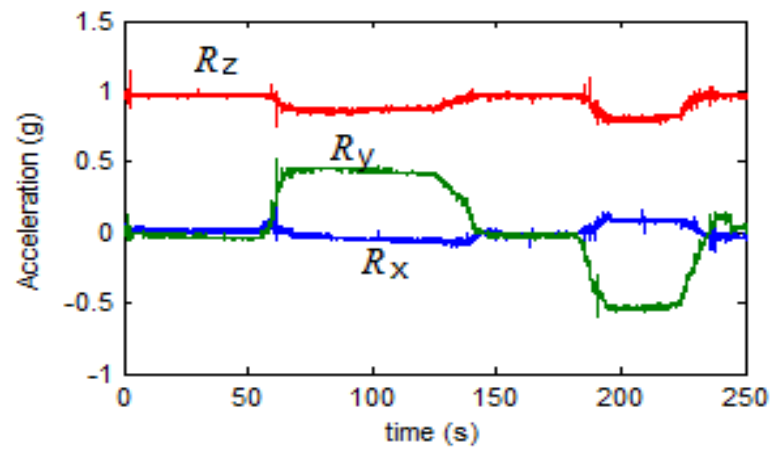

(a)

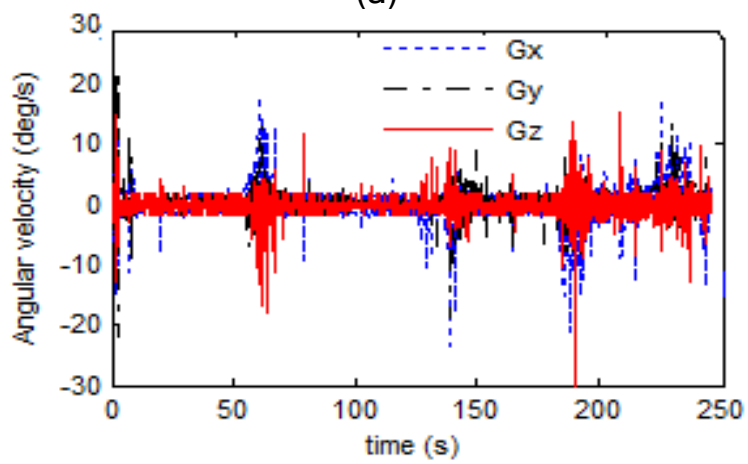

(b)

Figure 3. Euler Roll Angle Estimation using MEMS IMU, (a) MEMS MPU6050 TriAccelerometer Measurements, (b) MEMS MPU6050 Tri-gyroscope Measurements

Figure 3 (a) shows MEMS MPU6050 tri-accelerometer measurements for this experiment for total time of 250 seconds in the unit of ' $g$ ' whereas Figure 3 (b) shows MEMS MPU6050 tri-gyroscope measurements in unit of deg/second for the same. Similar pictorial view can be presented for pitch estimation test as well. Both ECA and GDCA use this data (from triaccelerometers and tri-gyroscopes) for attitude determination.

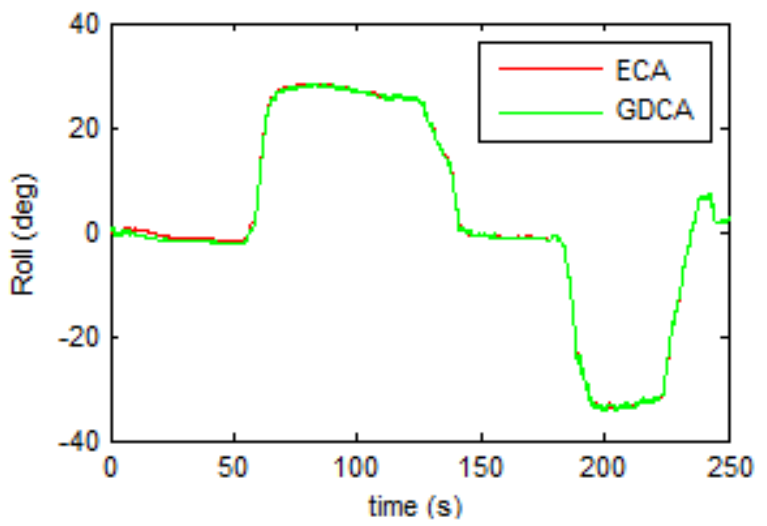

(a) 


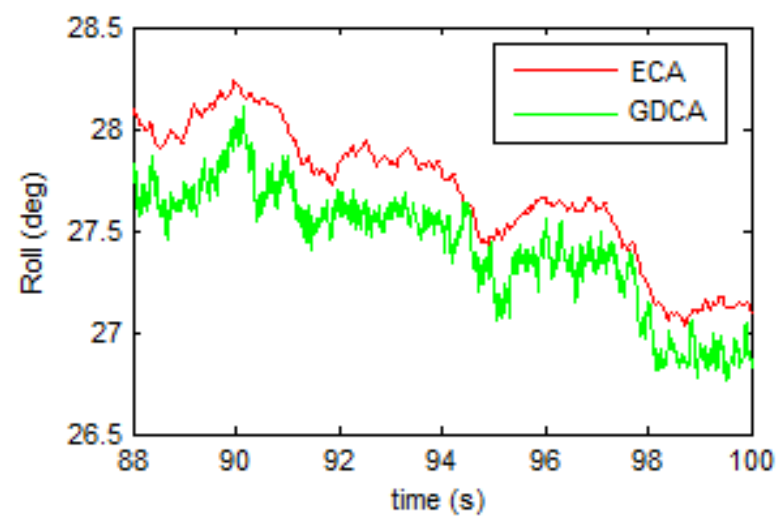

(b)

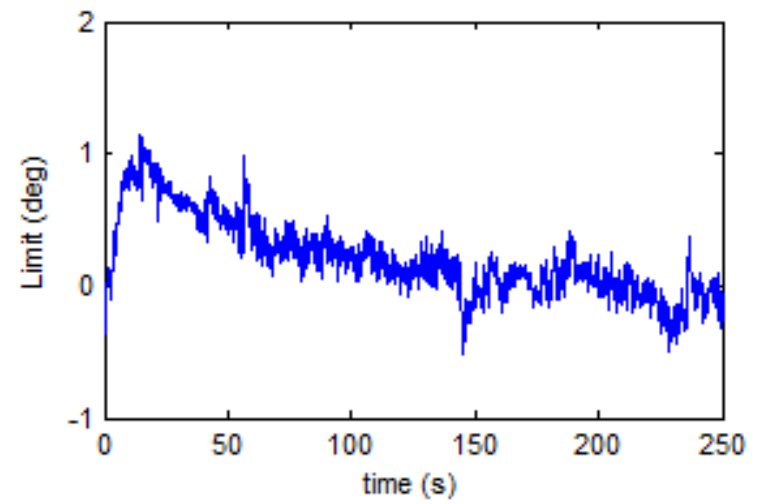

(c)

Figure 4. Euler Roll Angle Estimation using MEMS IMU, (a) Roll Estimation using ECA and GDCA, (b) Close View, (c) Estimation Difference between the Two Algorithms

Figure 4 (b) shows a close view of the roll estimation. It can be noted that both algorithms are in close proximity; ECA estimation being a bit more steady. Figure 4 (c) depicts the difference between the estimation by the two algorithms. It is evident that in steady state, the estimations from both algorithms lie within a limit of \pm 0.5 degrees. A similar approach was repeated for pitch and the result is shown in Figure 5. The two lines are hardly distinguishable, again strengthening the assumption that both algorithms closely follow each other by tuning the adjustable parameters. Again estimation from ECA seems more steady as compared to those from GDCA (Figure 4 (b)) and also the close proximity argument is strengthen by Figure 5(c). 
International Journal of Signal Processing, Image Processing and Pattern Recognition Vol. 8, No. 2 (2015)

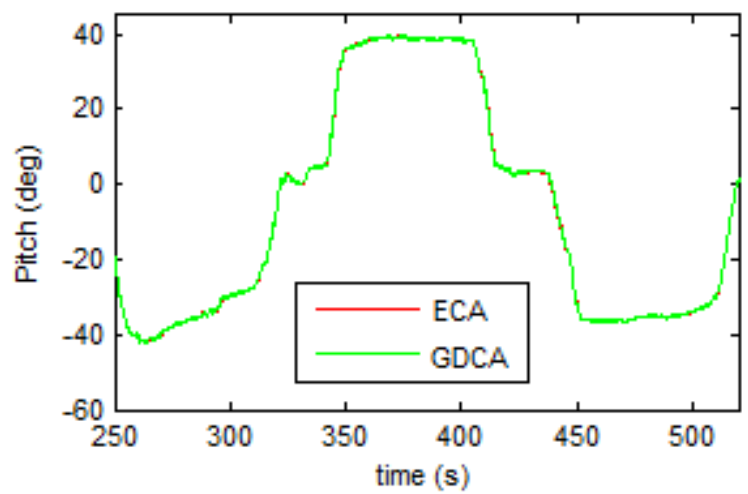

(a)

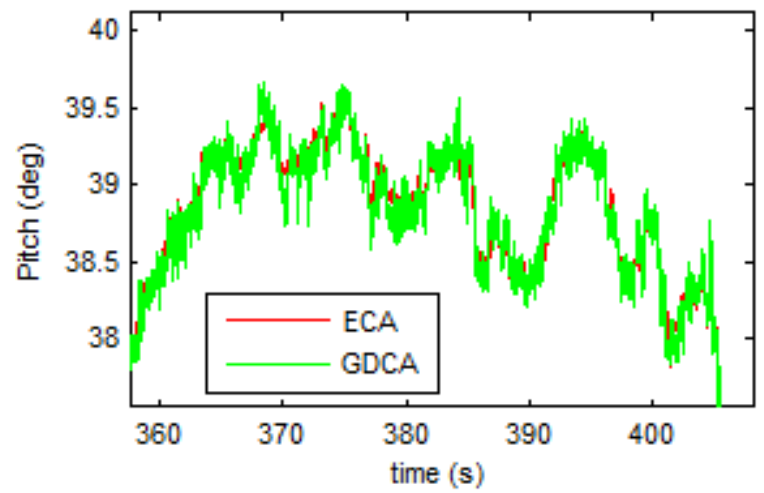

(b)

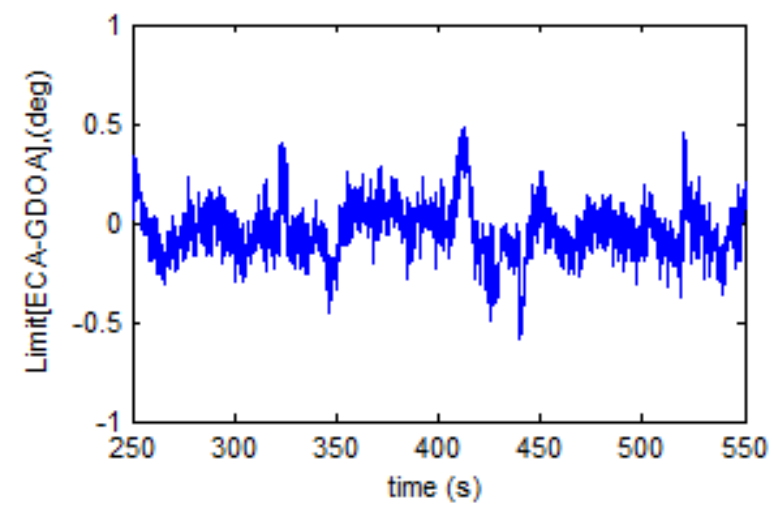

(c)

Figure 5. Euler Pitch Angle Estimation using MEMS IMU, (a) Pitch Estimation using ECA and GDCA, (b) Close View, (c) Estimation Difference between the Two Algorithms

For comparing the computational burden, execution time of the two algorithms was analyzed. Table 1 shows the results of the execution time required for the two algorithms in term of unit time (time required for GDCA algorithm to execute). From this point of view, the two algorithms computational simplicity is fairly the same. 
Table 1. Computation Complexity on Basis of Execution Time

\begin{tabular}{ccc}
\hline Algorithm & Execution time & Data \\
\hline ECA & 1.25 Unit & Simulated \&Experimental \\
GDCA & 1 Unit & Simulated \& Experimental \\
\hline
\end{tabular}

\section{Conclusion}

A comparative analysis was presented in this paper for the orientation problem using different approaches. Kalman filter and particle filter are considered the benchmark for position and attitude estimation, however, the associated computation burden in some circumstances are undesirable. Complementary filters are ideal in such situation. Both ECA and GDCA are effective and novel approaches in this regard. With the power of adjustable gain, these techniques find places in most of the real world applications. The evaluation of these filter results in identical outcome, however, ECA has a bit edge over GDCA partly because of higher accuracy and partly because of the two adjustable gains resulting in extra choices. Moreover, ECA is less sensitive to variation in filter gain in comparison with GDCA. Also, the computational complexity of the two algorithms is almost identical. Both techniques can be efficiently used in aided-INS system where less computation burden is of prime importance.

\section{References}

[1] X. Kong, "INS algorithm using quaternion model for low cost IMU", Robotics and Autonomous Systems, vol. 46, (2004), pp. 221-246.

[2] H. G. de Marina and F. J. Pereda, "UAV Attitude Estimation Using Unscented Kalman Filter and TRIAD", IEEE Transaction on Industrial Electronics, vol. 59, no. 11, (2012).

[3] E. Nebot and H. Durrant-Whyte, "Initial calibration and alignment of low cost inertial navigation units for land vehicle applications", J. of Robotics Sys, vol. 16, no. 2, (1999).

[4] C. Lee, S. Hong and W. Seong, "An Integrated DVL/IMU System for Precise Navigation of an Autonomous Underwater Vehicle", OCEANS Conf. Proceedings, (2003).

[5] S.-G. Kim, J. L. Crassidis and Y. Cheng, "Kalman Filtering for Relative spacecraft Attitude and Position Estimation" American Institute of Aeronautics and Astronautics, (2007).

[6] J. Wang and M. Garratt "Integration of GPS/INS/Vison Sensors to Navigate Unmanned Aerial Vehicles", The I.A. of the Phot., Remote Sen and Spat I.S., Beijing, vol. XXXVII, Part B1, (2008).

[7] O. J. Woodman, "An Introduction to Inertial Navigation", UCAM-CL-TR-696, University of Cambridge, (2007).

[8] R. Munguia and A. Grau, "A Practical Method for Implementing an Attitude and Heading Reference System”, International Journal of Advanced Robotic Systems, (2014).

[9] G. Grenon, P. E. An, M. Smith and J. Healey, "Enhancement of the Inertial Navigation System for the Morpheus Autonomous Underwater Vehicle," Journal of Oceanic Engineering, vol. 26, no. 4, (2001), pp. 548-560.

[10] X. Yun, G. C. Hernandez and E. R. Bachmann, "An Integrated GPS/INS Navigation System for Small AUVs Using an Asynchronous Kalman Filter," Proceeding of IEEE Symposium on Autonomous Underwater Vehicle Technology, (1998), pp. 43-49.

[11] D. Jurman, M. Jankovec and R. Kamnik, "Calibration and data fusion solution for the miniature attitude and heading reference system”, Sensors and Actuators A: Physical, vol. 138, Issue 2, (2007) August, pp. 411-420.

[12] L. Whitcomb, D. Yoerger and H. Singh, "Advances in Doppler-Based Navigation of Underwater Robotic Vehicles," Proceedings of IEEE International Conf. Robotics and Automation, vol. 1, (1999), pp. 399-406.

[13] P. Batista, C. Silvestre and P. Oliveira, "Low-cost Attitude and Heading Reference System: Filter design and experimental evaluation", Intl. Conf. on Robotics and Automation (ICRA), 2010 IEEE International Conference, (2010) May, pp. 2624-2629.

[14] M. Zimmerman and W. Sulzer, "High bandwidth orientation measurement and control based on complementary filtering," presented at the Symp. Robotics Control (SYROCO), Vienna, Austria, (1991).

[15] P. Corke, "An inertial and visual sensing system for a small autonomous Helicopter", Journal of Robotic Systems, vol. 21, Issue 2, (2004), pp. 43-51. 
[16] S. O. H. Madgwick and A. J. L. Harrison, "Estimation of IMU and MARG orientation using a gradient descent algorithm”, 2011 IEEE International Conference on Rehabilitation Robotics Rehab Week Zurich, ETH Zurich Science City, Switzerland, (2011) June 29 - July 1.

[17] T. S. Yoo and S. K. Hong, "Gain-Scheduled Complementary Filter Design for a MEMS Based Attitude and Heading Reference System”, Sensors, vol. 11, (2011), pp. 3816-3830, doi: 10.3390/s110403816.

[18] M. Wang and Y. C. Yang, "Adaptive filter for a miniature MEMS based attitude and heading reference system," presented in Pro IEEE Pos Loc and Nav Symp, Monterey, CA, USA, (2004) April.

[19] C. Coopmans, A. M. Jensen and Y. Chen, "Fractional-Order Complementary Filters for Small Unmanned Aerial System Navigation”, J. of intl. and robotic sys., vol. 73, Issue 1-4, (2014) January, pp. 429-453.

[20] M. Euston, P. Coote and R. Mahony, "A Complementary Filter for Attitude Estimation of a Fixed-Wing UAV”, IEEE/RSJ International Conference on Intelligent Robots and Systems, IROS (2008).

[21] M. D. Shuster, "A survey of attitude representations", The Journal of the Astronautical Sciences, vol. 41, no. 4, (1993), pp. 439-517.

[22] C. W. Kang and C. G. Park, "Attitude Estimation with Accelerometers and Gyros using Fuzzy Tuned Kalman filter", proc. Of EU cont. conf, Hungary, (2009).

[23] M. Pedley, "Tilt Sensing Using a Three-Axis Accelerometer", document number, AN3461, Rev. 6, 03/2013.

\section{Authors}
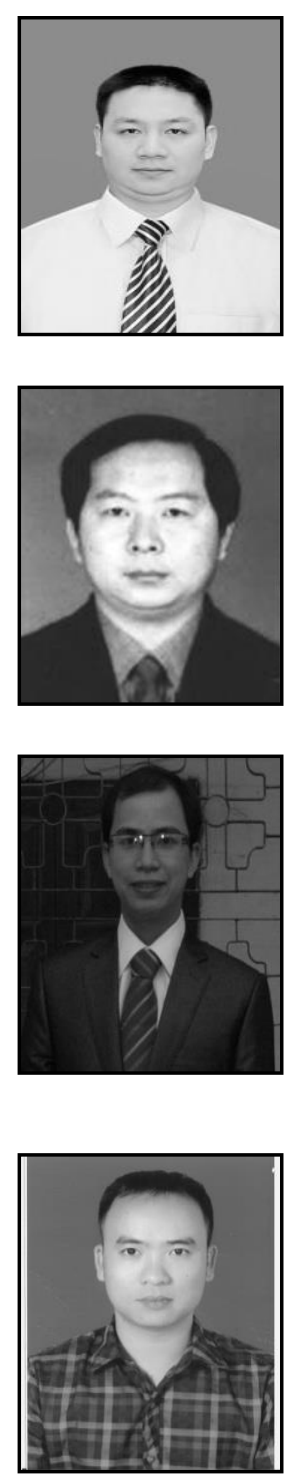

Dung Duong Quoc was born in 1981. He received the B.S., M.S. degrees from the Le Qui Don Technical University, Hanoi, Vietnam, in 2007 and 2010, respectively. Now he is pursuing Ph.D. degree in Harbin Institute of Technology (HIT), Harbin, China. His research interests include sensor technology, testing instruments, and signal processing.

Jin Wei Sun (M'09) was born in 1964. He received the M.S. degree in electrical engineering from Harbin Institude of Technology (HIT), Harbin, China, in 1990 and the Ph.D. degree in advanced system control and production from Saga University, Saga, japan, in 2000. He is currently a Professor with HIT. His research interests include sensor technology, testing instruments, and signal processing.

Van Nhu Le, Male, was born in 1982. He received the B.S degrees from the Le Quy Don Technical University, Hanoi, Vietnam, in 2007 and M.S dgree from Harbin Institute of Technology in 2012. Now he is pursuing Ph.D. degree in Research center for Space Optics Engineering, Harbin Institute of Technology, Harbin, China. He currently focuses image processing, optic image processing and design optic system.

Nguyen Ngoc Tan, Male, was born in 1986. He received B.S. degree from the Posts \& Telecommunication Institute of Technology, Hanoi, Vietnam in 2012 and M.S degree from the Le Quy Don Technical University, Hanoi, Vietnam in 2014. Now he is working for CMC Telecom Company, Phạm Hung Street, Caugiay district, Hanoi. He currently focuses on digital signal processing, signal sonar processing, polarimetric SAR, polarimetric SAR interferometry. 